\title{
Theoretical approaches to studying the single and simultaneous reactions in laminar flow-based membraneless fuel cells
}

\author{
WenYau Chen*, Falin Chen \\ Institute of Applied Mechanics, College of Engineering, National Taiwan University, No. 1, Sec. 4, Roosevelt Road, Taipei 10617, Taiwan \\ Received 8 June 2006; received in revised form 25 July 2006; accepted 25 July 2006 \\ Available online 2 October 2006
}

\begin{abstract}
Experiments with a laminar flow-based membrane-less fuel cell (LFMFC) have been conducted by many scientists. Choban et al. reported that the cell's performance is cathode limited. Accordingly, we have established half-cell models in our paper to study two types of redox reactions occurring at the cathode of the LFMFC without considering the fuel reaction. Our two-dimensional models are solved by using the spectral method where the eigenvalues are obtained by employing the Galerkin method. The similarity transform is applied to separate the concentrations of the oxidant and the intermediate product from their coupled boundary conditions. As shown in our results, the limiting average current density increases with the stoichiometry coefficient of electrons in the case of no intermediate product, yet the maximum electric power is independent of this coefficient. Given the concentrations of the oxidant and the intermediate product at the inlet end of the cell, we have obtained a condition increasing the current density. However, we also found the principle of generating a great deal of electric power by increasing the concentration of the intermediate product at the inlet end of the cell.
\end{abstract}

(C) 2006 Elsevier B.V. All rights reserved.

Keywords: Laminar flow-based membraneless micro fuel cell; Galerkin method; Butler-Volmer equation; Concentration boundary layer; Cathode electrode

\section{Introduction}

The possibility of generating electrical energy by continually feeding electrochemically active materials to a suitable cell has been investigated since 1839 by using platinum electrodes immersed in aqueous acid electrolytes. However, important progress in fuel cell technology dates from the early 1950s due to the requirements of the US Space program which has stimulated many researchers to seek electrical power that is clean, safe, efficient, economically feasible, and capable of quick start-up.

Many researchers have developed various fuel cells [1] for solving the two conventional problems in fuel cells: (1) slow electrochemical reaction rates result in low efficiency and (2) hydrogen has not become a readily available fuel source. On the basis of the electrolyte employed in the cell, there are five main types of fuel cells: proton exchange membrane (PEM), alkaline, phosphoric acid, molten carbonate, and solid oxide fuel cell. In a proton exchange membrane fuel cell (PEMFC), for preventing

\footnotetext{
* Corresponding author. Tel.: +886 2 33665693; fax: +886223639290.

E-mail address: wenyau2004@yahoo.com (W. Chen).
}

the mixing of fuels and oxidants, two electrode plates are bound to either side of the PEM that is used for transferring the protons from the anode to the cathode. Excellent reviews of the PEMFC up to mid 1990s were presented by Prater [2] and Gottesfeld [3]. The computational works on the PEMFC can be referred to the studies of Bernardi and Verbrugge [4,5], Springer et al. [6,7], Fuller and Newman [8], Nguyen and White [9], Gurau et al. [10], Yi and Nguyen [11,12], Um et al. [13], and Kulikovsky [14].

However, the PEM has two disadvantages: (1) it must be continuously hydrated to provide good transport of protons and (2) the fuel can diffuse through the PEM to the cathode, causing the problem of a mixed potential. To avoid these issues, Ferrigno et al. [15] and Choban et al. [16], respectively, developed the laminar flow-based membrane-less fuel cell (LFMFC) in which the fuel and oxidant aqueous solutions are pressuredriven from separate sources into a common channel. In this channel, these two streams remain laminar parallel flows with a liquid-liquid interface. As a result, the first disadvantage of using the PEMFC is naturally eliminated for the LFMFC because the liquid-liquid interface is essentially a non-drying membrane. Moreover, the problem of mixed potential can be effectively prevented because the LFMFC is normally operated at the Peclet 


\section{Nomenclature}

$C_{\text {Int }}$ non-dimensional concentration of the intermediate product

$C_{\text {Ox }}$ non-dimensional oxidant concentration

$\bar{C}_{\text {Ox }}$ and $\bar{C}_{\text {Int }}$ the inlet-end concentrations of the oxidant and the intermediate product in the case of Eqs. (47a) and (47b)

$C_{1} \quad$ inlet-end concentration of the oxidant in the case of Eq. (17)

CBL concentration boundary layer

$i_{\mathrm{av}} \quad$ average current density generated at the cathode electrode by the reaction of Eq. (17)

$i_{\text {av-1 }}$ and $i_{\text {av-2 }}$ average current densities generated at the cathode electrode by the reactions of Eqs. (47a) and $(47 \mathrm{~b})$, respectively

$i_{\text {av-net }}$ and $P_{\text {net }}$ net average current density and net average electric power generated at the cathode electrode through the simultaneous reactions of Eqs. (47a) and (47b)

$k_{\text {Int }}$ and $k_{\text {Int }}^{\prime}$ the reaction rate constants for the intermediate product in the anodic process of Eq. (47a) and the cathodic one of Eq. (47b), respectively.

$k_{\mathrm{Ox}}$ and $k_{\mathrm{Rd}}$ the reaction rate constants for the oxidant $(\mathrm{Ox})$ and the final product $(\mathrm{Rd})$

LFMFC laminar flow-based membraneless fuel cells

$n \quad$ the stoichiometry constant for the electron

$P \quad$ average electric power generated at the cathode electrode by the reaction of Eq. (17), defined as $\eta$ time $i_{\text {av }}$

$P e \quad$ Peclet number

PEM proton exchange membrane

PEMFC proton exchange membrane fuel cells

\section{Greek letters}

$\eta \quad$ difference between the non-dimensional electric potentials of the cathode electrode surface and the adjacent electrolyte solution

$\eta_{\text {eq }}$ the potential $\eta$ at the equilibrium state

number above 10,000 [16] for optimal performance and for reducing the crossover diffusions of fuel and oxidant. Recently, the principle of laminar parallel flows has been successfully applied to other microfluidic systems [17-20]. To date, a surge of studies has been reported on micro power sources for the increasing demands of small and often portable devices capable of operating in longer periods without recharging; e.g., cell phones, laptop computers.

Ismagilov et al. [21] investigated the crossover diffusion of electrolytes in parallel flows. They found the scaling laws for the crossover diffusion: the thickness of the crossover diffusion zone is scaled as the one-third power of $(D Z / U)$ near the boundary and as the one-half power in the middle of the channel, where $D$ is the diffusivity, $Z$ the distance along the channel, and $U$ is the averaged velocity. Put succinctly, the thickness of the crossover diffusion is determined only by the Peclet number. In summary, these scaling laws provide a basic understanding of the crossover diffusion, which is required to predict the resolutions of patterning and the fabrication with laminar flow [22] and is also necessary for the designs of diffusive T sensors [23] and other components in micro analytical systems [24].

Anisin et al. studied two-dimensional models for the laminar single gas layer confined between a rigid plate and an electrode [25]. The electric current, as shown in their results, increases with the gas velocity.

Bazylak et al. [26] used a three-dimensional fully numerical model to calculate the convective mass transport in the LFMFC. Employing the reaction kinetics, Bazylak et al. derived the boundary conditions for the fuel and the oxidant. Their results show that the fuel utilization can be increased from $8 \%$ to $23 \%$ but only $3 \%$ of mixing observed at the outlet is increased if the inlet-end velocity is decreased from 0.1 to $0.02 \mathrm{~m} \mathrm{~s}^{-1}$. However, based on the simulation results, their proposed tapered-electrode design for the LFMFC enables the fuel utilization to reach more than $50 \%$.

The Butler-Volmer equation [27] has been applied to calculate the current density generated by a single reaction. However, there are possibilities for two reactions to occur simultaneously and these reactions are linked by a common reactant. For example, the single reaction $\mathrm{O}_{2}+4 \mathrm{H}^{+}+4 \mathrm{e}^{-} \rightleftarrows 2 \mathrm{H}_{2} \mathrm{O}$ occurring at the cathode electrode can be regarded as the sum of two simultaneous reactions [28]:

$\mathrm{O}_{2}+2 \mathrm{H}^{+}+2 \mathrm{e}^{-} \rightleftarrows \mathrm{H}_{2} \mathrm{O}_{2}$

and

$\mathrm{H}_{2} \mathrm{O}_{2}+2 \mathrm{H}^{+}+2 \mathrm{e}^{-} \rightleftarrows 2 \mathrm{H}_{2} \mathrm{O}$

As seen, reactions (I) and (II) are not independent but linked by the intermediate product, hydrogen peroxide. Experiments show that the hydrogen peroxide is a relatively stable and detectable intermediate product because reaction (I) is generally inherently faster than (II). However, the more hydrogen peroxide arising, the less electric current generated by reaction (I), because increasing the chemical potential of the hydrogen peroxide can activate the anodic process of reaction (I). On the contrary, because some of the hydrogen peroxide that accumulates at the cathode electrode will diffuse away, as a result, the hydrogen peroxide in reaction (II) is reduced and hence less electric current is generated by this reaction. If such linked simultaneous reactions occur at the cathode electrode, the net current density generated at the cathode will be treated as the sum of those currents generated, respectively, from the simultaneous reactions [28-30].

In this paper we use theoretical approaches to studying the single and linked simultaneous reactions occurring at the cathode electrode of the LFMFC. The reason why we focus only on the cathode-side reactions is based on a report [16] which mentioned that the entire current density cannot increase significantly with the fuel concentration but can be limited obviously by the low oxidant concentration. The experiments [16] showed that the cell's performance was cathode limited. Accordingly, in this paper we are interested in developing two-dimensional half- 
cell models for studying the cases of single reaction and linked simultaneous reactions occurring at the cathode. We begin with the governing equations for the steady incompressible parallel flows. We solve the mass transport equations by means of the spectral method where the eigenvalues are numerically obtained by using the Galerkin method [31].

\section{Mathematical models}

\subsection{Continuous and momentum equations}

Fig. 1 is a two-dimensional schematic of the LFMFC, where the oxidant and fuel solutions are pressure-driven into a common channel and remain in laminar parallel flows with a liquid-liquid interface. The catalyst electrodes are installed at two boundary walls $y^{*}=-H^{*}$ and $H^{*}$. The oxidant and fuel solutions are mainly transported, respectively, in the cathode side (between $y^{*}=-H^{*}$ and 0 ) and in the anode side (between $y^{*}=0$ and $\left.H^{*}\right)$. These two streams are assumed to be fully developed steady incompressible high-Peclet-number parallel flows. The crossover diffusion will be considered in the mass transport equation. We assume constant fluidic properties and neglect the electrostatic force in the momentum equation by means of the electroneutrality assumption (see Chapter 11 of Ref. [28]).

Using the characteristic velocity, length, and pressure:

$$
\begin{aligned}
& u_{i}^{*}=\left(\frac{v^{*}}{H^{*}}\right) u_{i}, \\
& \left(x^{*}, y^{*}\right)=H^{*}(x, y), \\
& P_{i}^{*}=\rho_{i}^{*}\left(\frac{v^{*}}{H^{*}}\right)^{2} P_{i},
\end{aligned}
$$

in which $v^{*}$ is a characteristic kinematic viscosity, we can obtain the non-dimensional continuity and momentum equations for the parallel flows in the common channel:

$\frac{\partial u_{i}}{\partial x}=0$

$0=-\frac{\partial P_{i}}{\partial x}+v_{i} \frac{\partial^{2} u_{i}}{\partial y^{2}}$

$0=-\frac{\partial P_{i}}{\partial y}$

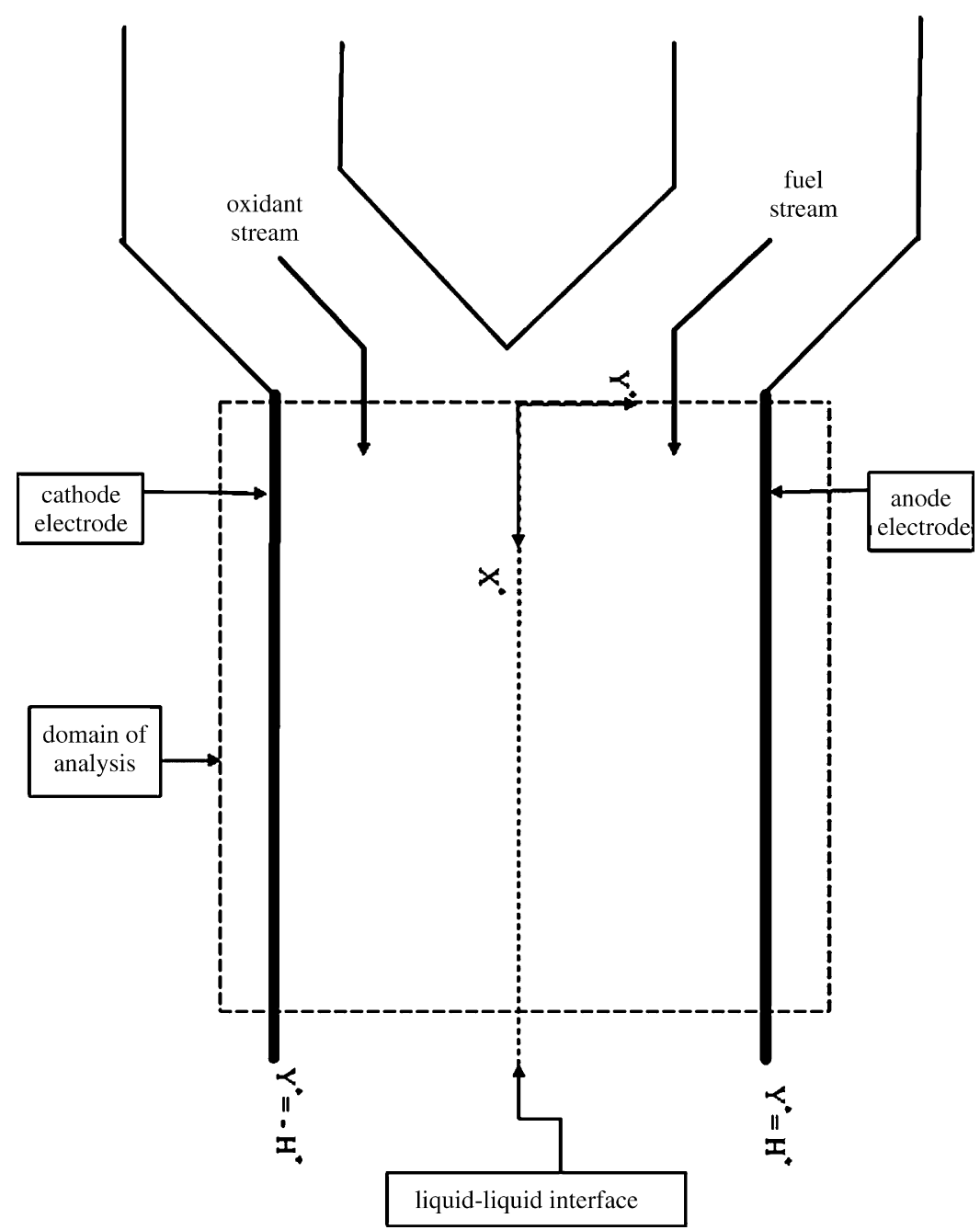

Fig. 1. Two-dimensional schematic of the LFMFC in which the oxidant and fuel streams are pressure-driven into a common channel and then remain in parallel flows with a liquid-liquid interface. 
where $v_{i}$ is defined as $v_{i}^{*} / \nu^{*}$. The subscript of $i=1$ represents the cathode-side stream that is transported between $y=-1$ and 0 . The anode-side stream is represented by $i=2$ and transported between $y=0$ and 1 . The solution to the velocity $u_{i}$ is a parabolic profile:

$u_{i}=-\bar{U}_{i}\left[y^{2}+A_{i} y+B_{i}\right]$

$\bar{U}_{i}=\frac{-\partial P_{i} / \partial x}{2 v_{i}}$

We use four boundary conditions to solve the undetermined coefficients in Eq. (7) for $i=1$ and 2: they are, respectively, continuous velocity and shear stress at $y=0$ plus no-slip conditions at $y= \pm 1$. Moreover, the $x$-direction pressure gradients in the cathode- and anode-side streams must be equal according to Eq. (6) and the dynamic boundary condition. As a result, we obtain:

$\frac{\partial P_{1}}{\partial x}=\frac{\partial P_{2}}{\partial x} \equiv \delta p$

$\bar{U}_{i} \equiv-\frac{\delta p}{2 \nu_{i}}$

$A_{1}=\frac{M\left(1-r_{\mathrm{u}}\right)}{M+r_{\mathrm{u}}}$

$A_{2}=\frac{1-r_{\mathrm{u}}}{M+r_{\mathrm{u}}}$

$B_{1}=-\frac{r_{\mathrm{u}}(1+M)}{M+r_{\mathrm{u}}}$

$B_{2}=-\frac{1+M}{M+r_{\mathrm{u}}}$

$M=\frac{\rho_{2}^{*}}{\rho_{1}^{*}}$

$r_{\mathrm{u}}=\frac{v_{1}^{*}}{v_{2}^{*}}$

\subsection{Mass transport models}

In this subsection, we introduce the half-cell models and theoretical approaches to studying the single and linked simultaneous reactions occurring at the cathode electrode. As described above, the fuel reaction is not considered because the cell's performance is cathode limited [16]. The stoichiometry coefficients of the oxidant, intermediate product, and the final product are assumed to be one. We assume high-Peclet-number flows for the reason described in the Introduction. In particular, in the high-Peclet-number flow, the $y$-direction gradient of the oxidant concentration near the cathode electrode becomes steeper, so a larger amount of the oxidant can diffuse to the electrode.

\subsection{1. $\operatorname{Part}(A)$}

$\mathrm{Ox}+2 \mathrm{mH}^{+}+2 n \mathrm{e}^{-} \rightleftarrows \mathrm{Rd}$

Eq. (17) is a single redox reaction where the notations Ox and $\mathrm{Rd}$ represent, respectively, the oxidant and the final product. A proton source, such as sulfuric acid, can be added to the cathodeside stream to provide a supply of protons for the consumption of protons at the cathode electrode [16,32,33]. In fact, the concentration of the sulfuric acid is very large as compared to that of the oxidant; the ratio between the concentrations of the sulfuric acid and the oxygen is 1000 as shown in the paper [16]. Therefore, in such a very acidic solution, the concentration of protons can be treated as a constant [28]. In this paper the final product is assumed to be pure liquid or solid, of which the activity is one. Electromigration is not considered for it could be small as compared to the convective transport in the high-Pecletnumber flow. Furthermore, due to the crossover diffusion, the $y$ variable of the oxidant concentration must range from -1 to 1 , so the oxidant will be transported by a different velocity profile as it diffuses across the liquid-liquid interface (see Eq. (7)). Accordingly, we can write down the non-dimensional mass transport equation and the boundary conditions for the oxidant as follows:

Pe $\tilde{u}_{i} \frac{\partial C_{\mathrm{Ox}}}{\partial x}=\frac{\partial^{2} C_{\mathrm{Ox}}}{\partial y^{2}}$

$\frac{\partial C_{\mathrm{Ox}}}{\partial y}=k_{\mathrm{Ox}} C_{\mathrm{Ox}} \mathrm{e}^{-2 \beta n \eta}-k_{\mathrm{Rd}} \mathrm{e}^{2 \beta^{\prime} n \eta}, \quad y=-1$

$\frac{\partial C_{\mathrm{Ox}}}{\partial y}=0, \quad y=1$

where

$\tilde{u}_{i} \equiv-\frac{1}{2 \nu_{i}}\left(y^{2}+A_{i} y+B_{i}\right)$

$P e \equiv \frac{-v^{*} \delta P}{D_{\mathrm{Ox}}^{*}}$

the superscript of star $(*)$ stands for dimensional terms and $C_{\mathrm{Ox}}$ is the non-dimensional oxidant concentration based on the characteristic concentration (one molarity). The notation $\mathrm{Pe}$ is the so-called Peclet number. We quantitatively describe the reaction of Eq. (17) by using the form of the Butler-Volmer equation as shown in Eq. (19) where $k_{\mathrm{Ox}}$ and $k_{\mathrm{Rd}}$ are, respectively, the nondimensional reaction rate constants for the oxidant and the final product. Although the inequality of $\beta+\beta^{\prime} \neq 1$, strictly speaking, holds for the processes involving more than one electron [34], we will assume that both $\beta$ and $\beta^{\prime}$ are equal to 0.5 in this paper. In Eq. (19) $\eta$ is a non-dimensional term defined by

$\eta \equiv \frac{F \eta^{*}}{R T}$

where $F$ is the Faraday number and $\eta^{*}$ is the difference between the physical electric potentials of the cathode electrode surface and the adjacent electrolyte solution. $D_{\mathrm{Ox}}^{*}$ is the dimensional diffusivity for the oxidant. Note that the activity of the final product, $\mathrm{Rd}$, is one. To solve for $C_{\mathrm{Ox}}$, we transform Eqs. (18)-(20) as follows:

$\operatorname{Pe} \tilde{u}_{i} \frac{\partial \tilde{C}_{\mathrm{Ox}}}{\partial x}=\frac{\partial^{2} \tilde{C}_{\mathrm{Ox}}}{\partial \tilde{y}^{2}}$ 
$\frac{\partial \tilde{C}_{\text {Ox }}}{\partial \tilde{y}}=\varepsilon \tilde{C}_{\text {Ox }}, \quad \tilde{y}=-2$

$\frac{\partial \tilde{C}_{\mathrm{Ox}}}{\partial \tilde{y}}=0, \quad \tilde{y}=0$

where

$\varepsilon \equiv k_{\mathrm{Ox}} \mathrm{e}^{-2 \beta n \eta}$

$\tilde{C}_{\mathrm{Ox}} \equiv C_{\mathrm{Ox}}-K^{-1} \mathrm{e}^{2 n \eta}$

$K^{-1} \equiv \frac{k_{\mathrm{Rd}}}{k_{\mathrm{Ox}}}$

$\tilde{y} \equiv y-1$

Let

$\tilde{C}_{\text {Ox }}=\sum_{k=1}^{N} \alpha_{k} \exp \left\{-\frac{\sigma_{k}}{P e} x\right\} \psi_{k}(\tilde{y})$

Substituting Eq. (31) into Eqs. (24)-(26), we obtain

$\frac{d^{2} \psi_{k}}{d \tilde{y}^{2}}+\sigma_{k} \tilde{u}_{i} \psi_{k}=0$

$\frac{\mathrm{d} \psi_{k}}{\mathrm{~d} \tilde{y}}=\varepsilon \psi_{k}, \quad$ at $\tilde{y}=-2$

$\frac{\mathrm{d} \psi_{k}}{\mathrm{~d} \tilde{y}}=0, \quad$ at $\tilde{y}=0$

We express $\psi_{k}$ by

$\psi_{k}=\sum_{n=1}^{N} a_{n, k} \phi_{n}(\tilde{y})$

To match the boundary conditions Eqs. (33) and (34), we may select

$\phi_{n}(\tilde{y})=\cos \left(\frac{\hat{\beta}_{n} \tilde{y}}{2}\right)$.

So $\hat{\beta}_{n}$ must satisfy

$\hat{\beta}_{n} \tan \hat{\beta}_{n}=2 \varepsilon$

The eigenvalues $\hat{\beta}_{n}$ can be solved numerically. By substituting Eq. (35) into Eq. (32), an eigenvalue equation based on the Galerkin method is obtained as follows, from which we can solve for $a_{n, k}$ and $\sigma_{k}$ :

$[\mathrm{H}]^{-1}[\mathrm{R}] \vec{a}_{k}=\sigma_{k} \vec{a}_{k}$

where

$\vec{a}_{k}=\left[a_{1, k}, a_{2, k}, \ldots, a_{N, k}\right]^{T}$

$[H]_{i, j}=\int_{-2}^{0} \tilde{u}_{i} \phi_{i} \phi_{j} \mathrm{~d} \tilde{y}$

$[R]_{i, j}=\left(\frac{\hat{\beta}_{n}}{2}\right)^{2} \delta_{i, j}, \quad i, j=1, \ldots, N$
Carrying out the computation of Eq. (38), we obtain one set of $\left\{a_{1, k}, a_{2, k}, \ldots, a_{N, k}\right\}$ for each $\sigma_{k}$ to compose the corresponding $\psi_{k}$ according to Eq. (35).

Let $C_{\mathrm{Ox}}$ at the inlet end of the common channel $(x=0)$ be given by

$C_{\mathrm{Ox}}= \begin{cases}0 & \text { for } \quad-1<\tilde{y}<0 \\ C_{1} & \text { for } \quad-2<\tilde{y}<1\end{cases}$

where $C_{1}$ is the inlet-end concentration of the oxidant, so the inlet-end condition for $\tilde{C}_{\mathrm{Ox}}$ can be found from Eq. (28):

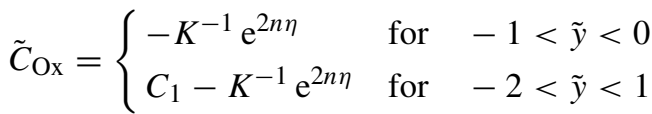

By means of the orthogonality, the coefficients $\alpha_{k}$ in Eq. (31) can be solved by

$\alpha_{k}=\frac{\int_{-2}^{0} \tilde{C}_{\mathrm{Ox}}(x=0) \tilde{u}_{i} \psi_{k} \mathrm{~d} \tilde{y}}{\int_{-2}^{0} \tilde{u}_{i} \psi_{k}^{2} \mathrm{~d} \tilde{y}}$

After that, $\tilde{C}_{\text {Ox }}$ can be obtained from Eq. (31), and hence $C_{\mathrm{Ox}}$ is solved. Now, the local current density generated at the cathode electrode can be calculated by substituting the $C_{\mathrm{Ox}}$ into Eq. (19). Let the current density be rendered non-dimensional by using the characteristic current density $\left(F D_{\mathrm{Ox}}^{*} 1\right.$ molarity) $/ H^{*}$. We define an average current density as

$\left.i_{\mathrm{av}} \equiv \frac{1}{L} \int_{0}^{L} 2 n\left(k_{\mathrm{Ox}} C_{\mathrm{Ox}} \mathrm{e}^{-2 \beta n \eta}-k_{\mathrm{Rd}} \mathrm{e}^{2 \beta n \eta}\right)\right|_{\tilde{y}=-2} \mathrm{~d} x$

where $L$ is the non-dimensional length of the cathode electrode. According to Eq. (19), we have

$i_{\mathrm{av}}=\left.\frac{2 n}{L} \int_{0}^{L} \frac{\partial C_{\mathrm{Ox}}}{\partial \tilde{y}}\right|_{\tilde{y}=-2} \mathrm{~d} x$.

\subsection{2. $\operatorname{Part}(B)$}

$\mathrm{Ox}+\mathrm{mH}^{+}+n \mathrm{e}^{-} \rightleftarrows \mathrm{Int}$,

Int $+\mathrm{mH}^{+}+n \mathrm{e}^{-} \rightleftarrows \mathrm{Rd}$

Now, we consider two simultaneous reactions linked by an intermediate product (Int) as shown in Eqs. (47a) and (47b), and their sum is exactly the single reaction of Eq. (17), for instance, the case of $\mathrm{O}_{2}+4 \mathrm{H}^{+}+4 \mathrm{e}^{-} \rightleftarrows 2 \mathrm{H}_{2} \mathrm{O}$. In this paper we assume the ratio between the diffusivities of the oxidant and the intermediate product is roughly equal to one, because the diffusivity for most electrolytes in dilute solutions is in the order of $10^{-9} \mathrm{~m}^{2} \mathrm{~s}^{-1}$ [35]. In the following, we present the mass transport equations and the boundary conditions for the concentrations of the oxidant and the intermediate product $\left(C_{\mathrm{Ox}}\right.$ and $\left.C_{\text {Int }}\right)$ on a basis of the same characteristic scales and assumptions as those used in part (A):

$\operatorname{Pe} \tilde{u}_{i} \frac{\partial}{\partial x}\left[\begin{array}{c}C_{\mathrm{Ox}} \\ C_{\text {Int }}\end{array}\right]=\frac{\partial^{2}}{\partial \tilde{y}^{2}}\left[\begin{array}{c}C_{\mathrm{Ox}} \\ C_{\text {Int }}\end{array}\right]$

$\frac{\partial C_{\mathrm{Ox}}}{\partial \tilde{y}}=k_{\mathrm{Ox}} C_{\mathrm{Ox}} \mathrm{e}^{-\beta_{\mathrm{a}} n \eta}-k_{\mathrm{Int}} C_{\mathrm{Int}} \mathrm{e}^{\beta_{\mathrm{a}}^{\prime} n \eta}, \quad \tilde{y}=-2$ 
$-\frac{\partial C_{\text {Int }}}{\partial \tilde{y}}=k_{\mathrm{Ox}} C_{\mathrm{Ox}} \mathrm{e}^{-\beta_{\mathrm{a}} n \eta}-k_{\mathrm{Int}} C_{\mathrm{Int}} \mathrm{e}^{\beta_{\mathrm{a}}^{\prime} n \eta}-k_{\text {Int }}^{\prime} C_{\text {Int }} \mathrm{e}^{-\beta_{\mathrm{b}} n \eta}$

$$
+k_{\mathrm{Rd}} \mathrm{e}^{\beta_{\mathrm{b}}^{\prime} n \eta}, \quad \tilde{y}=-2
$$

$\frac{\partial}{\partial \tilde{y}}\left[\begin{array}{c}C_{\text {Ox }} \\ C_{\text {Int }}\end{array}\right]=0, \quad \tilde{y}=0$

where $P e$ and $\tilde{u}_{i}$ are defined in Eqs. (21) and (22). We can combine Eqs. (49a) and (49b) into:

$$
\begin{aligned}
\frac{\partial}{\partial \tilde{y}}\left[\begin{array}{c}
C_{\text {Ox }} \\
C_{\text {Int }}
\end{array}\right]= & {\left[\begin{array}{cc}
k_{\text {Ox }} \mathrm{e}^{-\beta_{\mathrm{a}} n \eta} & -k_{\text {Int }} \mathrm{e}^{\beta_{\mathrm{a}}^{\prime} n \eta} \\
-k_{\text {Ox }} \mathrm{e}^{-\beta_{\mathrm{a}} n \eta} & k_{\text {Int }} \mathrm{e}^{\beta_{\mathrm{a}}^{\prime} n \eta}+k_{\text {Int }}^{\prime} \mathrm{e}^{-\beta_{\mathrm{b}} n \eta}
\end{array}\right]\left[\begin{array}{c}
C_{\text {Ox }} \\
C_{\text {Int }}
\end{array}\right] } \\
& +\left[\begin{array}{c}
0 \\
-k_{\mathrm{Rd}} \mathrm{e}^{\beta_{\mathrm{b}}^{\prime} n \eta}
\end{array}\right]
\end{aligned}
$$

Note that each electron transfer coefficient in Eq. (51), just as like as $\beta$ and $\beta^{\prime}$ in Eq. (19), is assumed to be 0.5 . The notations $k_{\text {Int }}$ and $k_{\text {Int }}^{\prime}$ represent the reaction rate constants of the intermediate product in the anodic process of Eq. (47a) and in the cathodic process of Eq. (47b), respectively. However, we can rewrite Eq. (51) as follows:

$$
\begin{aligned}
\frac{\partial}{\partial \tilde{y}}\left[\begin{array}{c}
C_{\text {Ox }} \\
C_{\text {Int }}
\end{array}\right]= & \tilde{\varepsilon}\left[\begin{array}{cc}
1 & -\tilde{\kappa}_{1} \mathrm{e}^{n \eta} \\
-1 & \tilde{\kappa}_{1} \mathrm{e}^{n \eta}+\tilde{\kappa}_{2}
\end{array}\right]\left[\begin{array}{c}
C_{\text {Ox }} \\
C_{\text {Int }}
\end{array}\right] \\
& +\left[\begin{array}{c}
0 \\
-k_{\text {Rd }} \mathrm{e}^{\beta n \eta}
\end{array}\right]
\end{aligned}
$$

where

$$
\begin{aligned}
& \tilde{\varepsilon} \equiv k_{\mathrm{Ox}} \mathrm{e}^{-\beta n \eta} \\
& \tilde{\kappa}_{1} \equiv \frac{k_{\mathrm{Int}}}{k_{\mathrm{Ox}}} \\
& \tilde{\kappa}_{2} \equiv \frac{k_{\mathrm{Int}}^{\prime}}{k_{\mathrm{Ox}}} \\
& \beta \equiv 0.5
\end{aligned}
$$

Define the matrix in Eq. (52) as $[A]$ :

$[A] \equiv\left[\begin{array}{ll}A_{11} & A_{12} \\ A_{21} & A_{22}\end{array}\right] \equiv\left[\begin{array}{cc}1 & -\tilde{\kappa}_{1} \mathrm{e}^{n \eta} \\ -1 & \tilde{\kappa}_{1} \mathrm{e}^{n \eta}+\tilde{\kappa}_{2}\end{array}\right]$

Using the similarity transform, we have

$[A]=[P][\Lambda][P]^{-1}$

and

$[\Lambda] \equiv\left[\begin{array}{cc}\lambda_{1} & 0 \\ 0 & \lambda_{2}\end{array}\right]$

where $\lambda_{1}$ and $\lambda_{2}$ are the distinct eigenvalues of $[A]$. The first and second columns in the $[P]$ matrix are, respectively, the eigen- vectors corresponding to $\lambda_{1}$ and $\lambda_{2}$; that is,

$$
\left[\begin{array}{ll}
A_{11} & A_{12} \\
A_{21} & A_{22}
\end{array}\right]\left[\begin{array}{l}
P_{1, m} \\
P_{2, m}
\end{array}\right]=\lambda_{m}\left[\begin{array}{l}
P_{1, m} \\
P_{2, m}
\end{array}\right], \quad m=1,2
$$

If we define

$$
\left[\begin{array}{l}
S_{1} \\
S_{2}
\end{array}\right] \equiv[P]^{-1}\left[\begin{array}{c}
C_{\mathrm{Ox}} \\
C_{\text {Int }}
\end{array}\right]
$$

$\left[\begin{array}{l}f_{1} \\ f_{2}\end{array}\right] \equiv[P]^{-1}\left[\begin{array}{c}0 \\ -k_{\mathrm{Rd}} \mathrm{e}^{\beta n \eta}\end{array}\right]$

and

$\left[\begin{array}{l}t_{1} \\ t_{2}\end{array}\right] \equiv\left[\begin{array}{l}S_{1} \\ S_{2}\end{array}\right]+\left[\begin{array}{l}f_{1} /\left(\tilde{\varepsilon} \lambda_{1}\right) \\ f_{2} /\left(\tilde{\varepsilon} \lambda_{2}\right)\end{array}\right]$

then we can rewrite Eqs. (48), (50) and (52), respectively, as follows:

$$
\begin{aligned}
& \operatorname{Pe} \tilde{u}_{i} \frac{\partial}{\partial x}\left[\begin{array}{l}
t_{1} \\
t_{2}
\end{array}\right]=\frac{\partial^{2}}{\partial \tilde{y}^{2}}\left[\begin{array}{l}
t_{1} \\
t_{2}
\end{array}\right] \\
& \frac{\partial}{\partial \tilde{y}}\left[\begin{array}{l}
t_{1} \\
t_{2}
\end{array}\right]=0, \quad \tilde{y}=0 \\
& \frac{\partial}{\partial \tilde{y}}\left[\begin{array}{l}
t_{1} \\
t_{2}
\end{array}\right]=\left[\begin{array}{l}
\tilde{\varepsilon} \lambda_{1} t_{1} \\
\tilde{\varepsilon} \lambda_{2} t_{2}
\end{array}\right] \equiv\left[\begin{array}{l}
\tilde{\varepsilon}_{1} t_{1} \\
\tilde{\varepsilon}_{2} t_{2}
\end{array}\right], \quad \tilde{y}=-2
\end{aligned}
$$

As seen, both $t_{1}$ and $t_{2}$ are not coupled as $C_{\mathrm{Ox}}$ and $C_{\mathrm{Int}}$ in Eq. (52). The conditions of $t_{1}$ and $t_{2}$ at the inlet end of the common channel $(x=0)$ can be found through Eqs. (61)-(63) where the inlet-end conditions of $C_{\mathrm{Ox}}$ and $C_{\mathrm{Int}}$ are given as follows:

$\left.C_{\text {Ox }}\right|_{x=0}= \begin{cases}0 & \text { for } \quad-1<\tilde{y}<0 \\ \bar{C}_{\text {Ox }} & \text { for } \quad-2<\tilde{y}<1\end{cases}$

$\left.C_{\text {Int }}\right|_{x=0}= \begin{cases}0 & \text { for } \quad-1<\tilde{y}<0 \\ \bar{C}_{\text {Int }} & \text { for } \quad-2<\tilde{y}<1\end{cases}$

Therefore, we can separately solve Eqs. (64)-(66) for $t_{1}$ and $t_{2}$ by means of the same method for solving Eqs. (24)-(26), and hence $C_{\mathrm{Ox}}$ and $C_{\mathrm{Int}}$ can be obtained through the reverse transform from Eq. (61)-(63). Now, we can calculate the net average current density which is the sum of two average current densities generated, respectively, by the simultaneous reactions of Eqs. (47a) and (47b). They are expressed as

$\left.i_{\text {av }-1} \equiv \frac{1}{L} \int_{0}^{L} n\left(k_{\mathrm{Ox}} C_{\mathrm{Ox}} \mathrm{e}^{-\beta n \eta}-k_{\text {Int }} C_{\text {Int }} \mathrm{e}^{\beta n \eta}\right)\right|_{\tilde{y}=-2} \mathrm{~d} x$

$\left.i_{\text {av }-2} \equiv \frac{1}{L} \int_{0}^{L} n\left(k_{\text {Int }}^{\prime} C_{\text {Int }} \mathrm{e}^{-\beta n \eta}-k_{\text {Rd }} \mathrm{e}^{\beta n \eta}\right)\right|_{\tilde{y}=-2} \mathrm{~d} x$

where $i_{\mathrm{av}-1}$ and $i_{\mathrm{av}-2}$ are non-dimensional quantities based on the same characteristic current density used in part (A). Thus the net average current density is defined by

$i_{\mathrm{av}-\mathrm{net}}=i_{\mathrm{av}-1}+i_{\mathrm{av}-2}$. 


\section{Results and discussion}

In this section we present the modeling results of both current density and electric power as a function of potential $\eta$ for the reactions in parts (A) and (B). As defined in Eq. (23), $\eta$ is the difference between the non-dimensional electric potentials of the cathode electrode surface and the adjacent electrolyte solution. For saving on computation, the potential $\eta$ is restricted to the range from 0 to less than 30 . Consequently, the conversion by using Eq. (23) shows that the range of the physical potential $\eta^{*}$ is within $0-0.775 \mathrm{~V}$ for room temperature $(R T / F=0.02585 \mathrm{~V})$. Unless otherwise specified, the following parameters are fixed: (1) the number of expansion terms in Eq. (31) $N=150$, similarly for $t_{1}$ and $t_{2}$ expansions, (2) the Peclet number, $P e=10^{6}$, (3) $M$ and $r_{\mathrm{u}}$ in Eqs. (15) and (16) are set to be one and (4) all electron transfer coefficients in Eqs. (19), (49a) and (49b) are specified as 0.5 .

\subsection{Results for part (A)}

Fig. 2a shows the average current density $i_{\text {av }}$ versus the potential $\eta$ for the reaction of Eq. (17) for four cases of $k_{\mathrm{Rd}}$, in which we specify three parameters: the ratio $k_{\mathrm{Ox}}$ to $k_{\mathrm{Rd}}(\mathrm{K})$, stoichiometry coefficient $n$, and the inlet-end concentration of the oxidant $\left(C_{1}\right)$. As mentioned above, we set the range of $\eta$ as from 0 to 20 $\left(\eta^{*}=0-0.517 \mathrm{~V}\right)$. As a result, considering the clarity of Fig. 2a, we present only four cases of $k_{\mathrm{Rd}}$ within this potential range. Those curves for $k_{\mathrm{Rd}}$ larger than $10^{-8}$ and less than $10^{-19}$ are so close to the curves of $k_{\mathrm{Rd}}=10^{-8}$ and $10^{-19}$ that they are not dis-

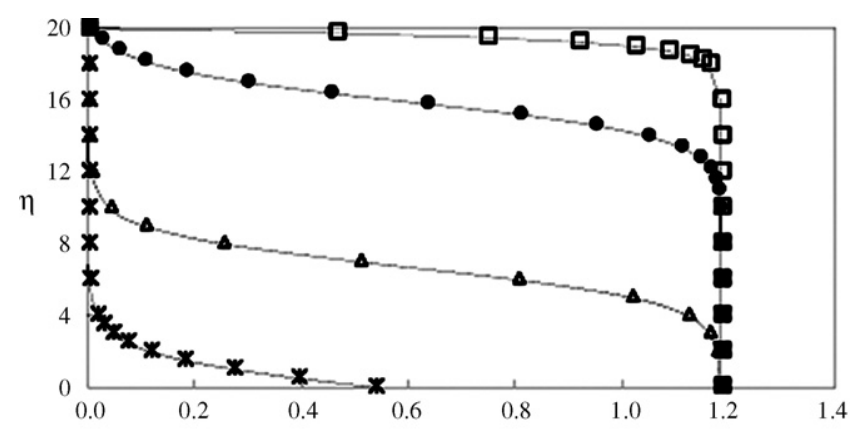

(a)
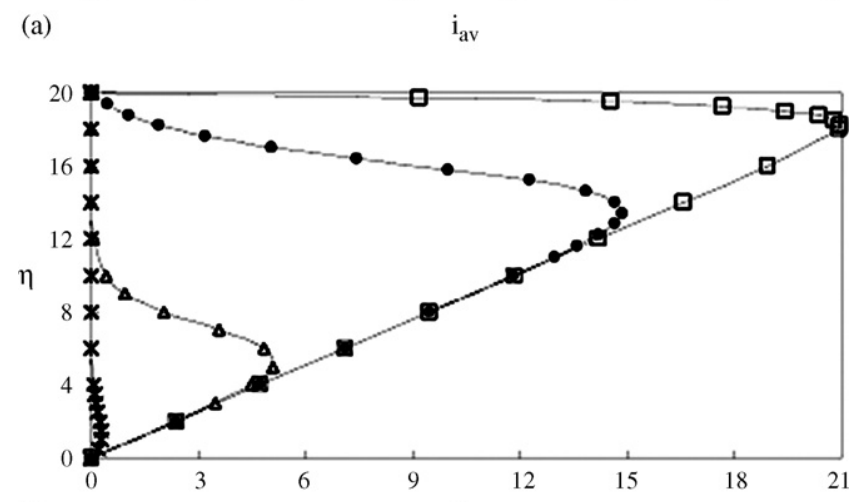

(b)

$\mathrm{P}$

Fig. 2. Average current density $i_{\text {av }}$ and average electric power $P$ vs. the potential $\eta$ where $K=e^{40} \times 10^{3}, n=1$, and $C_{1}=10^{-3}$. The symbol of rectangular box stands for the case of $k_{\mathrm{Rd}}=10^{-8}$; black circle: $k_{\mathrm{Rd}}=10^{-12}$; triangle: $k_{\mathrm{Rd}}=10^{-16}$; star: $k_{\mathrm{Rd}}=10^{-19}$. played in the figure; accordingly, we choose $10^{-8}$ and $10^{-19}$ as the range of $k_{\mathrm{Rd}}$ in Fig. 2a for pure graphic consideration. In the equilibrium, the current densities generated by the cathodic and the anodic processes of Eq. (17) are equal such that the derivative of $C_{\mathrm{Ox}}$ respective to $y$ at the cathode electrode is equal to 0 according to Eq. (19), whereby we can infer $C_{\mathrm{Ox} \mid y=-1}=C_{1}$. Accordingly, we can obtain from Eq. (19) the potential $\eta$ at the equilibrium state:

$\eta_{\mathrm{eq}}=\frac{\ln \left(k_{\mathrm{Ox}} C_{1} / k_{\mathrm{Rd}}\right)}{2 n}=\frac{\ln \left(C_{1} K\right)}{2}=20$

which is independent of $k_{\mathrm{Rd}}$ as shown in Fig. 2a. Furthermore, we combine Eqs. (30), (45) and (72) to rewrite the average current density as

$$
\begin{aligned}
i_{\mathrm{av}}= & \frac{1}{L} \int_{0}^{L} 2 n k_{\mathrm{Rd}} \mathrm{e}^{2 n \beta \eta_{\mathrm{eq}}} \\
& \times\left(\frac{C_{\mathrm{Ox}, y=-1}}{C_{1}} \mathrm{e}^{-2 \beta n\left(\eta-\eta_{\mathrm{eq}}\right)}-\mathrm{e}^{2 \beta n\left(\eta-\eta_{\mathrm{eq}}\right)}\right) \mathrm{d} x
\end{aligned}
$$

Apparently, the potential $\eta$ must be decreased much further from the equilibrium-state value in order to generate appreciable current density in the case of smaller $k_{\mathrm{Rd}}$, because we know that the smaller the $k_{\mathrm{Rd}}$ and $k_{\mathrm{Ox}}$, the less the exchange current density. For instance, to generate the average current density of 0.4 , the reduction of $\eta$ is found to be $1 \%$ and $97 \%$ of $\eta_{\mathrm{eq}}$ for the cases of $k_{\mathrm{Rd}}=10^{-8}$ and $10^{-19}$, respectively. Now, we define an average electric power by $P=\eta i_{\text {av }}$. Fig. $2 \mathrm{~b}$ shows that the maximum $P$ decreases as $k_{\mathrm{Rd}}$ is diminished.

In contrast to Fig. 2 where $k_{\mathrm{Rd}}$ is varied yet $\mathrm{n}$ is fixed, Fig. 3a shows the effects of $n$ on the cell's performance, where $k_{\mathrm{Rd}}$ is fixed, while $C_{1}$ and $K$ remain the same as in Fig. 2. As seen, the equilibrium-state value of the potential $\eta$ decreases with increasing $n$ : $\eta_{\mathrm{eq}}=20,10$, and 6.67 for $n=1,2$, and 3, respectively. However, this property can be checked by substituting these $n$ values into Eq. (72). On the contrary, the limiting average current density increases with $n$. In fact, as the potential $\eta$ is lowered to a certain extent, the cathodic process of Eq. (17) overwhelms the anodic one such that the oxidant at the cathode electrode surface $(y=-1)$ is nearly depleted. Consequently the $y$-direction gradient of the oxidant concentration at $y=-1$ reaches the limit, which can be estimated as follows:

$$
\left.\frac{\partial C_{\mathrm{Ox}}}{\partial y}\right|_{y=-1} \cong \frac{C_{\mathrm{Ox}, \text { bulk region }}-C_{\mathrm{Ox}, y=-1}}{d_{\mathrm{c}}} \cong \frac{C_{\mathrm{Ox}, \text { bulk region }}}{d_{\mathrm{c}}}=\frac{C_{1}}{d_{\mathrm{c}}}
$$

where $d_{\mathrm{c}}$ is the so-called thickness of the concentration boundary layer (CBL) of the oxidant. This thickness is proportional to the reverse of the square root of the Peclet number according to Eq. (18), yet independent of n. In the situation of Eq. (74), the $y$ direction diffusion of the oxidant reaches the maximum so the average current density will reach its limit as well. We combine Eqs. (30), (46) and (74) to express this limiting average current density as

$$
i_{\mathrm{av}}=\left.\frac{2 n}{L} \int_{0}^{L} \frac{\partial C_{\mathrm{Ox}}}{\partial y}\right|_{y=-1} \mathrm{~d} x \cong \frac{2 n C_{1}}{L} \int_{0}^{L} \frac{\mathrm{d} x}{d_{\mathrm{c}}}
$$




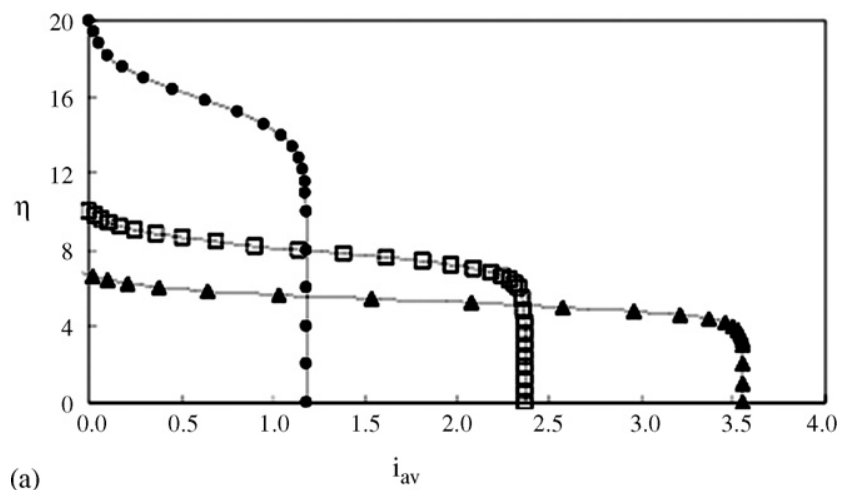

(a)

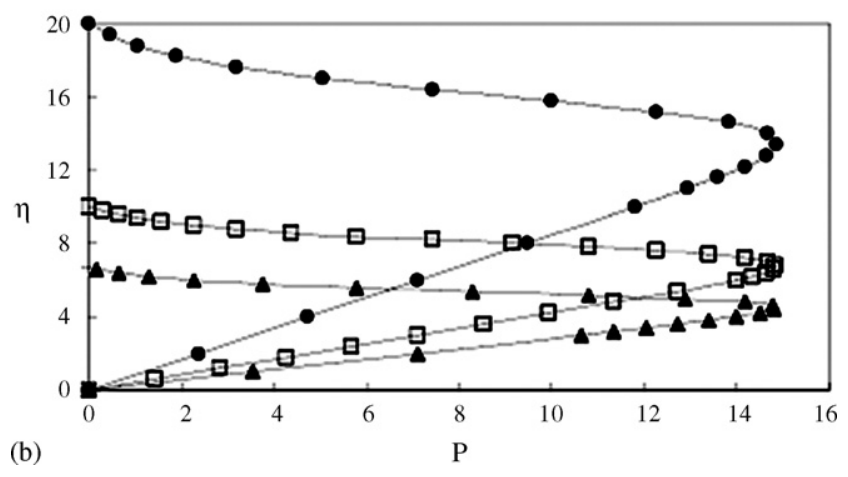

Fig. 3. Average current density $i_{\mathrm{av}}$ and average electric power $P$ vs. the potential $\eta$ where $K=e^{40} \times 10^{3}, k_{\mathrm{Rd}}=10^{-12}$, and $C_{1}=10^{-3}$. The symbol of black circle stands for the case of $n=1$; rectangular box: $n=2$; black triangle: $n=3$.

One can see that this limiting value is theoretically linear with $n$ because $d_{\mathrm{c}}$ is independent of $n$. As shown in Fig. 3a, the limiting $i_{\text {av }}$ is found to be $1.18,2.37$, and 3.55 for $n=1,2$, and 3 , respectively. Obviously their ratio is nearly $1: 2: 3$, which coincides with Eq. (75). Next, we display in Fig. 3b the results of the average electric power $P$ calculated for those three cases of $n$ in Fig. 3a. However, the maximum $P$ is about the same, nearly independent of $n$. Moreover, the potential $\eta$ at which the maximum $P$ is generated is found to be $13.4,6.8$, and 4.5 for $n=1,2$, and 3 , respectively. The ratio among these potentials is about $1: 1 / 2: 1 / 3$.

\subsection{Results for part $(B)$}

When studying the simultaneous reactions of Eqs. (47a) and (47b), we are especially interested in the effects of the intermediate product on the cell's performance. Consequently, in this subsection our discussion is focused on the theoretical understanding of the principles: how the current density is changed with the reaction rate constants of the intermediate product $\left(k_{\text {Int }}\right.$ and $k_{\text {Int }}^{\prime}$ ) and changed with the concentration of the intermediate product. In order to qualitatively describe the effects of $k_{\text {Int }}$ and $k_{\text {Int }}^{\prime}$, in Figs. 4 and 5, we investigate three sets of $k_{\text {Int }}$ and $k_{\text {Int }}^{\prime}$ in which we specify the $k_{\mathrm{Ox}}$ and $k_{\mathrm{Rd}}$, and the inlet-end concentrations of the oxidant and the intermediate product $\left(\bar{C}_{\text {Ox }}\right.$ and $\left.\bar{C}_{\text {Int }}\right)$. Next, in Figs. 6 and 7, we choose three cases of $\bar{C}_{\text {Int }}$ to study the effects of the concentration of the intermediate product on the cell's performance. It should be noticed that the ranges of $k_{\text {Int }}$ and $k_{\text {Int }}^{\prime}$ (both are from $10^{-8}$ to $10^{8}$ ) are specified for pure
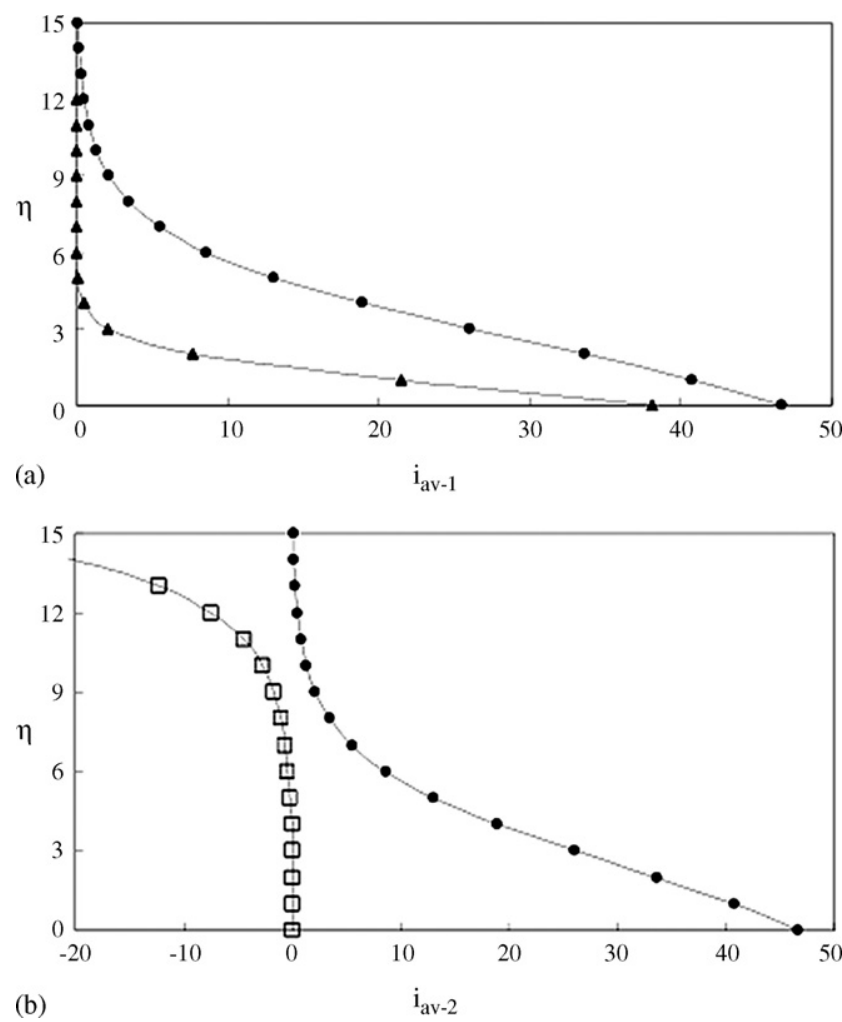

Fig. 4. Average current densities $i_{\mathrm{av}-1}$ and $i_{\mathrm{av}-2}$ vs. the potential $\eta$ for $n=1$, $k_{\mathrm{Ox}}=10^{2}, k_{\mathrm{Rd}}=10^{-5}, \bar{C}_{\mathrm{Ox}}=0.1$, and $\bar{C}_{\mathrm{Int}}=0$. The symbol of black circle stands for the case of $k_{\text {Int }}=10^{-8}$ and $k_{\text {Int }}^{\prime}=10^{8}$; black triangle; $k_{\text {Int }}=10^{8}$ and $k_{\text {Int }}^{\prime}=10^{8}$; rectangular box: $k_{\text {Int }}=10^{-8}$ and $k_{\text {Int }}^{\prime}=10^{-8}$.
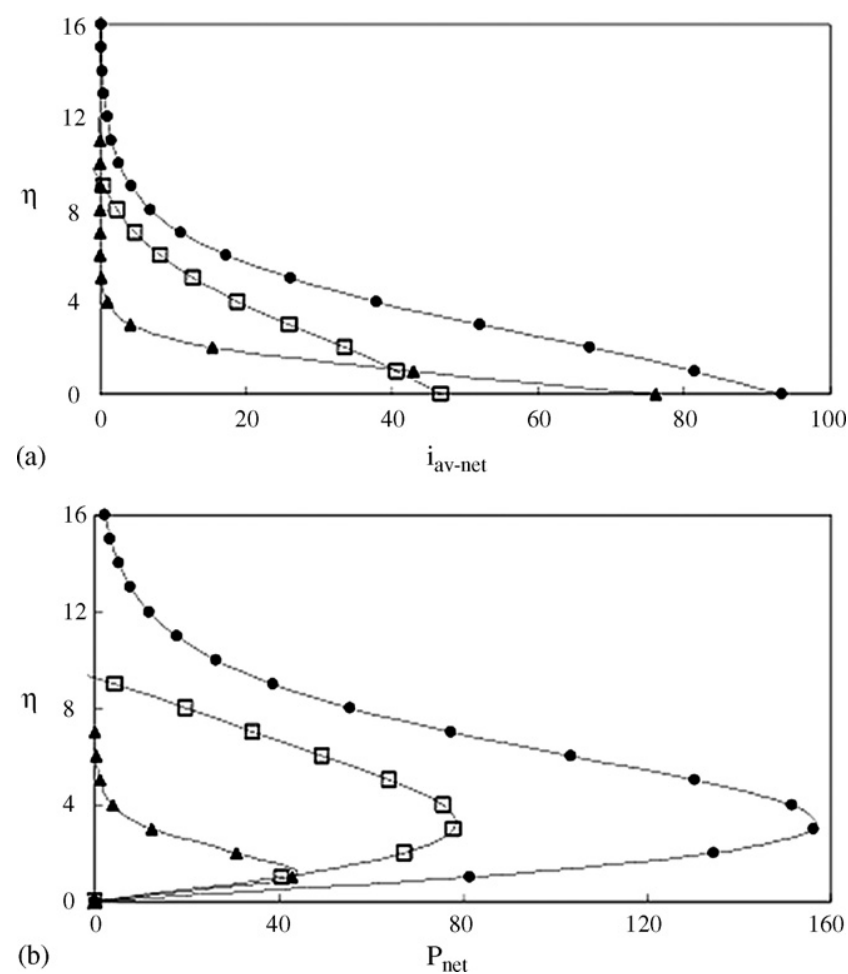

Fig. 5. Net average current density $i_{\text {av-net }}$ and net average electric power $P_{\text {net }}$ vs. the potential $\eta$ for $n=1, k_{\mathrm{Ox}}=10^{2}, k_{\mathrm{Rd}}=10^{-5}, \bar{C}_{\mathrm{Ox}}=0.1$, and $\bar{C}_{\mathrm{Int}}=0$. The symbol of black circle stands for the case of $k_{\mathrm{Int}}=10^{-8}$ and $k_{\text {Int }}^{\prime}=10^{8}$; black triangle: $k_{\mathrm{Int}}=10^{8}$ and $k_{\mathrm{Int}}^{\prime}=10^{8}$; rectangular box: $k_{\mathrm{Int}}=10^{-8}$ and $k_{\mathrm{Int}}^{\prime}=10^{-8}$. 

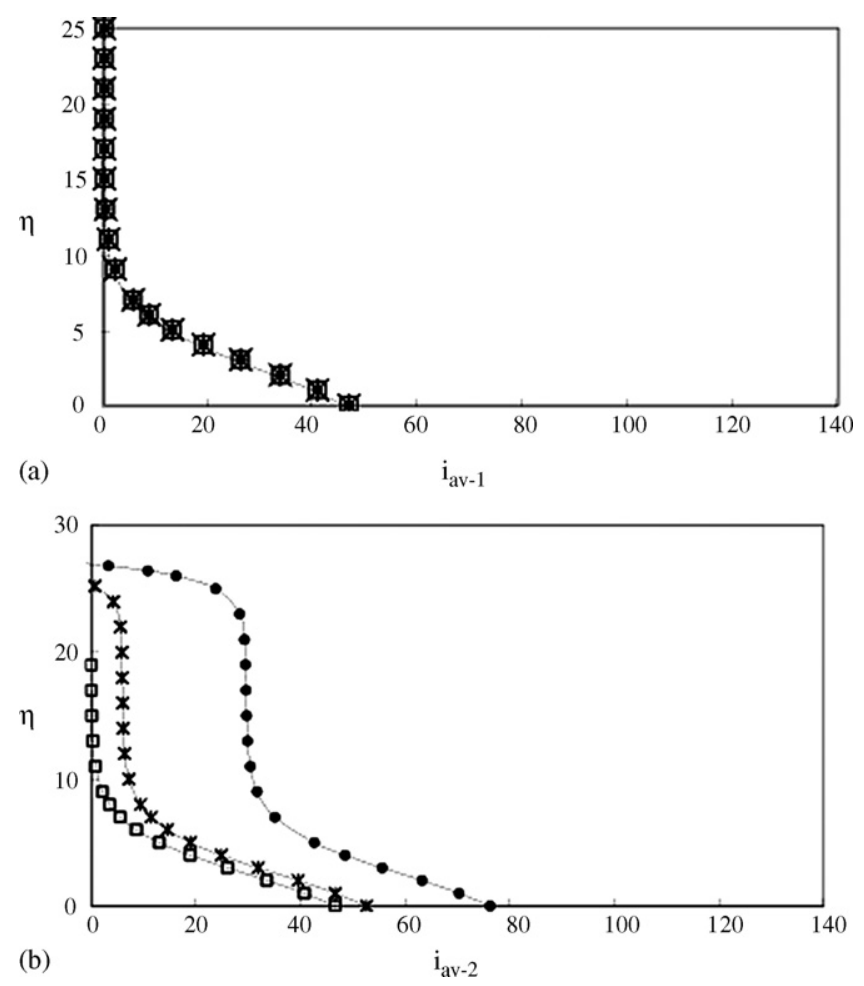

Fig. 6. Average current densities $i_{\mathrm{av}-1}$ and $i_{\mathrm{av}-2}$ vs. the potential $\eta$ for $n=1$, $k_{\mathrm{Ox}}=10^{2}, k_{\mathrm{Rd}}=10^{-5}, k_{\mathrm{Int}}=10^{-8}, k_{\mathrm{Int}}^{\prime}=10^{8}$, and $\bar{C}_{\mathrm{Ox}}=0.1$. The symbol of rectangular box stands for the case of $\bar{C}_{\text {Int }}=0$; star: $\bar{C}_{\text {Int }}=0.01$; black circle: $\bar{C}_{\text {Int }}=0.05$.

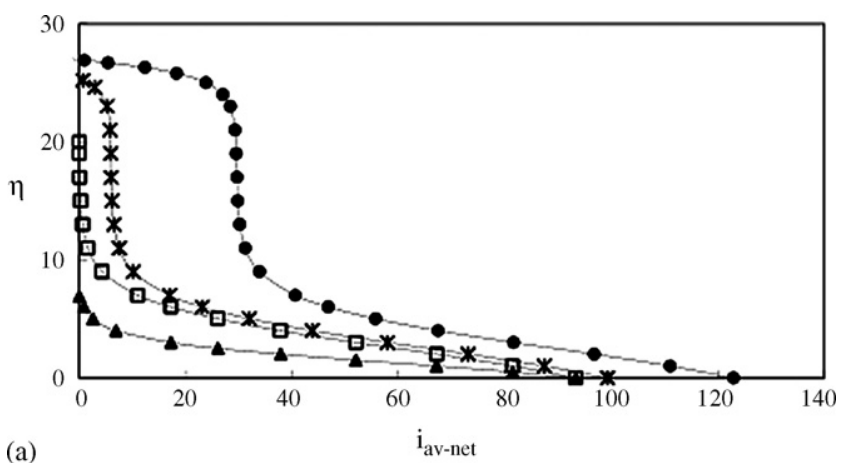

(a)

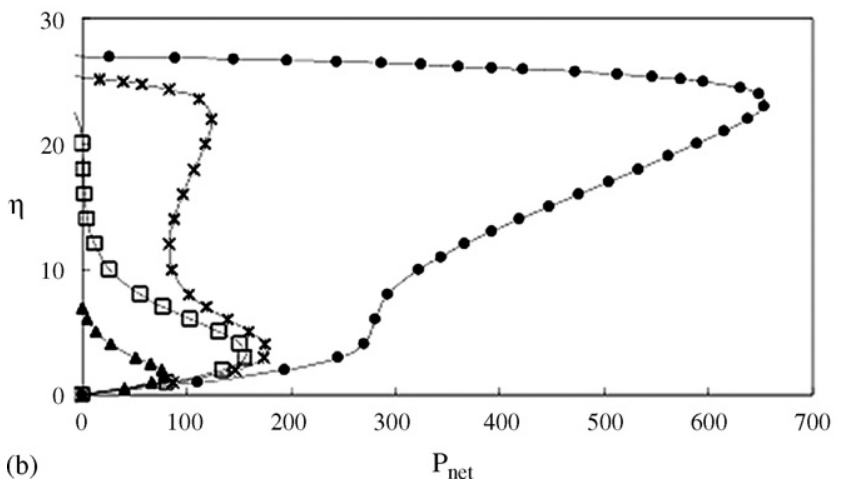

Fig. 7. Net average current density $i_{\text {av-net }}$ and net average electric power $P_{\text {net }}$ vs. the potential $\eta$ where $n=1, k_{\mathrm{Ox}}=10^{2}, k_{\mathrm{Rd}}=10^{-5}, k_{\mathrm{Int}}=10^{-8}, k_{\mathrm{Int}}^{\prime}=10^{8}$, and $\bar{C}_{\mathrm{Ox}}=0.1$. The symbol of rectangular box stands for the case of $\bar{C}_{\mathrm{Ox}}=0$; star: $\bar{C}_{\text {Int }}=0.01$; black circle: $\bar{C}_{\text {Int }}=0.05$. The symbol of black triangle represents the $i_{\text {av }}$ and $P$ calculated for the single reaction of Eq. (17). computational consideration, not for any particular intermediate species, because the main purpose in this subsection is to qualitatively describe the effect of the intermediate product on the cell's performance.

First, we display in Fig. 4a the results of calculated $i_{\mathrm{av}-1}$ by using Eq. (69) for $k_{\text {Int }}=10^{-8}$ and $10^{8}$, whereas $k_{\text {Int }}^{\prime}$ is fixed at $10^{8}$. It indicates that $i_{\mathrm{av}-1}$ becomes larger in the case of smaller $k_{\text {Int }}$. In contrast to Fig. $4 \mathrm{a}$, Fig. $4 \mathrm{~b}$ shows the $i_{\mathrm{av}-2}$ calculated by using Eq. (70) for $k_{\text {Int }}^{\prime}=10^{-8}$ and $10^{8}$, where $k_{\text {Int }}$ is fixed at $10^{-8}$. As seen, the $i_{\mathrm{av}-2}$ increases obviously with $k_{\text {Int }}^{\prime}$. Yet, it must be noticed that $i_{\mathrm{av}-2}$ is almost 0 or negative in the case of $k_{\mathrm{Int}}^{\prime}=10^{-8}$ because this $k_{\text {Int }}^{\prime}$ is a great deal smaller than the specified value of $k_{\mathrm{Rd}}$ and also because the $\bar{C}_{\text {Int }}$ is 0 such that the reaction of Eq. (47b) is dominated by the anodic process especially when the potential $\eta$ is enhanced. Accordingly, we infer, it is favorable to increasing the net average current density $i_{\text {av-net }}$ when both $k_{\mathrm{Ox}} / k_{\mathrm{Int}}$ and $k_{\mathrm{Int}}^{\prime} / k_{\mathrm{Rd}}$ are very large. To prove this, we present

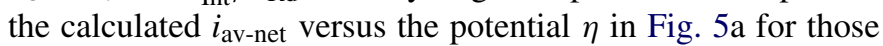
cases in Fig. 4. As shown, the results agree with our inference. On the contrary, the case of larger $k_{\text {Int }}$ coupled with smaller $k_{\text {Int }}^{\prime}$ will be more adverse to the generation of current density, which is not our interest and not presented in Fig. 5. In addition, we display in Fig. $5 \mathrm{~b}$ the net average electric power $P_{\text {net }}$ defined by $i_{\text {av-net }} \eta$. In summary, at given $\bar{C}_{\mathrm{Ox}}$ and $\bar{C}_{\text {Int }}$, simultaneously increasing the ratios $k_{\mathrm{Ox}} / k_{\mathrm{Int}}$ and $k_{\mathrm{Int}}^{\prime} / k_{\mathrm{Rd}}$ will be favorable to the enhancements of the net average current density and the electric power.

To more understand the effect of the intermediate product on the cell's performance, in addition to researching the effects of $k_{\text {Int }}$ and $k_{\text {Int }}^{\prime}$, we also want to study the effect of the concentration of the intermediate product. To carry out this work, we select the optimal case in Fig. 5 where $k_{\text {Int }}=10^{-8}$ and $k_{\text {Int }}^{\prime}=10^{8}$ because, as described in the last paragraph, this case is favorable to the generations of current density and electric power. Furthermore, we set $\bar{C}_{\text {Int }}=0,0.01$, and 0.05 , respectively. First, as shown in Fig. 6a, the calculated $i_{\mathrm{av}-1}$ by using Eq. (69) is about the same among these three cases of $\bar{C}_{\text {Int }}$ because the given ratio $k_{\mathrm{Ox}} / k_{\text {Int }}$ is so large that the second term in the integration of Eq. (69), as compared to the first term, is almost neglected for $\bar{C}_{\text {Int }}$ less than 0.05. Moreover, as displayed in Fig. 6b, the $i_{\mathrm{av}-2}$ increases remarkably because the specified value of $k_{\mathrm{Int}}^{\prime} / k_{\mathrm{Rd}}$ is so large that slightly increasing the $\bar{C}_{\text {Int }}$ can obviously enlarge the first term in the integration of Eq. (70). Accordingly, we infer, only when the ratios $k_{\mathrm{Ox}} / k_{\mathrm{Int}}$ and $k_{\mathrm{Int}}^{\prime} / k_{\mathrm{Rd}}$ are simultaneously very large will the net average current density obviously increase as $\bar{C}_{\text {Int }}$ is increased. To prove this, as defined in Fig. 5, the net average current density and the net average electric power for those cases in Fig. 6 are calculated and exhibited in Fig. 7a and $\mathrm{b}$, respectively. As seen, both $i_{\text {av-net }}$ and $P_{\text {net }}$ obviously increase with $\bar{C}_{\text {Int }}$. In particular, the maximum $P_{\text {net }}$ changes from 156 to 653 as $\bar{C}_{\text {Int }}$ is slightly increased from 0 to 0.05 . Checking Fig. 6, one can see that the evident increases in $i_{\text {av-net }}$ and $P_{\text {net }}$ are due to the changes in $i_{\mathrm{av}-2}$. Finally, we present in Fig. 7a and b the $i_{\mathrm{av}}$ and $P$ (black-triangle symbol) calculated for the single reaction of Eq. (17) to compare with the $i_{\text {av-net }}$ and $P_{\text {net }}$ at the same values of $n, k_{\mathrm{Ox}}, k_{\mathrm{Rd}}$, and the inlet-end concentration of the oxidant. As shown from the result of comparison, adding more intermediate 
product into the linked simultaneous reactions of Eqs. (47a) and (47b) can help us obtain higher power than that obtained though the single reaction of Eq. (17) as long as the ratios $k_{\mathrm{Ox}} / k_{\mathrm{Int}}$ and $k_{\text {Int }}^{\prime} / k_{\mathrm{Rd}}$ are very large.

\section{Conclusions}

Two-dimensional half-cell models were established for studying the current densities and electric power generated through the reactions of Eqs. (17), (47a) and (47b) that occur at the cathode of the LFMFC. The parabolic solutions to the fully developed steady incompressible high-Peclet-number parallel flows were obtained without considering the electrostatic forces. Mass transport by electromigration was neglected as compared to the convective transport because we assumed high-Pecletnumber flows in the study. We focused on the cathode-side redox reactions without considering the fuel reaction because a report [16] mentioned that the cell was cathode limited. We used the Butler-Volmer equation to describe the boundary conditions of the concentrations of the oxidant and the intermediate product at the cathode electrode. We solved the mass transport equations by means of the spectral method where the eigenvalues were numerically obtained through the Galerkin method.

For the single reaction of Eq. (17), given ratio $k_{\mathrm{Ox}} / k_{\mathrm{Rd}}$ and the inlet-end concentration of the oxidant, the potential $\eta$ at the equilibrium state does not change with $k_{\mathrm{Rd}}$ if $\mathrm{n}$ is fixed, but equilibrium-state $\eta$ decreases with increasing $n$ if $k_{\mathrm{Rd}}$ is fixed. The latter can be checked by substituting these $n$ values into Eq. (72). In addition, in the limiting condition, the average current density is linear with $n$, while the maximum average electric power is almost independent of $n$. However, the potential $\eta$ at which the maximum average electric power is generated in the cases of $n=1,2$, and 3 has the ratio nearly equal to $1: 1 / 2: 1 / 3$.

In the linked simultaneous reactions of Eqs. (47a) and (47b), the $i_{\mathrm{av}-1}$ becomes larger in the case of smaller $k_{\text {Int }}$ while $i_{\mathrm{av}-2}$ increases with $k_{\text {Int }}^{\prime}$. At given $\bar{C}_{\text {Ox }}$ and $\bar{C}_{\text {Int }}$, simultaneously increasing the ratios $k_{\mathrm{Ox}} / k_{\mathrm{Int}}$ and $k_{\mathrm{Int}}^{\prime} / k_{\mathrm{Rd}}$ is favorable to the enhancements of the net average current density and the net average electric power. However, the $i_{\mathrm{av}-1}$ is about the same for $\bar{C}_{\text {Int }}$ less than 0.05 if $k_{\mathrm{Ox}} / k_{\text {Int }}$ is very large. Nevertheless, the $i_{\mathrm{av}-2}$ can increase remarkably by slightly increasing the $\bar{C}_{\text {Int }}$ provided that the ratio $k_{\mathrm{Int}}^{\prime} / k_{\mathrm{Rd}}$ is very large. In summary, given the same values of $n, k_{\mathrm{Ox}}, k_{\mathrm{Rd}}$, and the inlet-end concentration of the oxidant, by adding a great deal of the intermediate product into the oxidant solution, one can obtain more power through the linked simultaneous reactions of Eqs. (47a) and (47b) than that obtained though the single reaction of Eq. (17) as long as the ratios $k_{\mathrm{Ox}} / k_{\mathrm{Int}}$ and $k_{\mathrm{Int}}^{\prime} / k_{\mathrm{Rd}}$ are very large.

\section{References}

[1] J. Larminie, A. Dicks, Fuel Cell Systems Explained, John Wiley, New York, 2000.
[2] K.B. Prater, J. Power Sources 51 (1994) 129.

[3] S. Gottesfeld, in: C. Tobias (Ed.), Advances in Electrochemical Science and Engineering, vol. 5, John Wiley, New York, 1997, p. 195.

[4] D.M. Bernardi, M.W. Verbrugge, AIChE J. 37 (1991) 1151-1163.

[5] D.M. Bernardi, M.W. Verbrugge, J. Electrochem. Soc. 139 (1992) 2477-2491.

[6] T.E. Springer, T.A. Zawodinski, S. Gottesfeld, J. Electrochem. Soc. 138 (1991) 2334-2342.

[7] T.E. Springer, M.S. Wilson, S. Gottesfeld, J. Electrochem. Soc. 140 (1993) 3513-3526.

[8] T.F. Fuller, J.J. Newman, J. Electrochem. Soc. 140 (1993) 12181225.

[9] T.V. Nguyen, R.E. White, J. Electrochem. Soc. 140 (1993) 2178 2186.

[10] V. Gurau, H. Liu, S. Kakac, AIChE J. 44 (1998) 2410-2422.

[11] J.S. Yi, T.V. Nguyen, J. Electrochem. Soc. 145 (1998) 1149-1159.

[12] J.S. Yi, T.V. Nguyen, J. Electrochem. Soc. 146 (1999) 38-45.

[13] S. Um, C.Y. Wang, K.S. Chen, J. Electrochem. Soc. 147 (2000) 44854490.

[14] A.A. Kulikovsky, J. Electrochem. Soc. 150 (2003) A1432-A1439.

[15] R. Ferrigno, A.D. Stroock, T.D. Clark, M. Mayer, G.M. Whitesides, J. Am. Chem. Soc. 124 (2002) 12930-12931.

[16] E.R. Choban, L.J. Markoski, A. Wieckowski, P.J.A. Kenis, J. Power Sources 128 (2004) 54-60.

[17] M.A. Burns, B.N. Johnson, S.N. Brahmasandra, K. Handique, J.R. Webster, M.K.T.S. Sammarco, P.M. Man, D. Jones, D. Heldsinger, C.H. Mastrangelo, D.T. Burke, Science 282 (1998) 484-487.

[18] B.H. Wiegl, J. Kriebel, K.J. Mayes, T. Bui, P. Yager, Acta 131 (1999) $75-83$.

[19] K. Macounova, C.R. Cabrera, M.R. Holl, P. Yager, Anal. Chem. 72 (2000) $3745-3751$

[20] K. Macounova, C.R. Cabrera, P. Yager, Anal. Chem. 73 (2001) 1627 1633.

[21] R.F. Ismagilov, A.D. Stroock, P.J.A. Kenis, G.M. Whitesides, Appl. Phys. Lett. 76 (2000) 2376-2378.

[22] P.J.A. Kenis, R.F. Ismagilov, G.M. Whitesides, Science 285 (1999) $83-85$.

[23] B.H. Weigl, P. Yager, Science 283 (1999) 346-347.

[24] G. Blankenstein, U.D. Larsen, Biosens. Bioelectron. 13 (1998) $427-$ 438.

[25] A.V. Anisin, I. Davydov, A.V. Kondrashenko, Russian J. Electrochem. 39 (2003) 898

[26] A. Bazylak, S. David, D. Ned, J. Power Sources 143 (2005) $57-$ 66.

[27] C.H. Hamann, A. Hamnett, W. Vielstich, Electrochemistry, Wiley, New York, 1998, pp. 143-186.

[28] J. Newmann, K.E. Thomas-Alyea, Electrochemical Systems, Wiley, New Jersey, 2004, pp. 203-232.

[29] K.J. Vetter, Electrochemical Kinetics, Academic Press, New York, 1967, pp. 732-747.

[30] M.G. Fontana, N.D. Greene, Corrosion Engineering, McGraw-Hill, New York, 1967.

[31] B.A. Finlayson, The Method of Weighted Residuals and Variational Principles, Academic Press, New York, 1972.

[32] S. Hasegawa, K. Shimotani, K. Kishi, H. Watanabe, Electrochem, Solid State Lett. 8 (2005) A119-A121.

[33] J.L. Cohen, D.J. Volpe, D.A. Westly, A. Pechenik, H.D. Abruna, Langmuir 21 (2005) 3544-3550.

[34] D.R. Crow, Principles and Applications of Electrochemistry, 4th ed., T.J. Press, Padstow, Cornwall, 1994, pp. 129-143.

[35] E.L. Cussler, Diffusion: Mass Transfer in Fluid Systems, 2nd ed., Cambridge University Press, New York, 1997. 